\title{
Bladder endometriosis complicated with cystitis glandularis mimicking recurrence of uterine cervical cancer: the unique value of transvaginal ultrasound
}

\section{Ruifeng Liu, Yu Xia}

Ultrasound Department, Peking Union Medical College Hospital, Chinese Academy of Medical Sciences and Peking Union Medical College, Beijing, China

\section{To the Editor,}

We read the article by Brătilă et al with great interest [1]. They proposed an innovative method using sonovaginography with ultrasound gel to better diagnose deep infiltrating endometriosis. In the practice routine, we realized that transvaginal ultrasound (TVU) actually pos- sesses unique value in assessing unusual cases of bladder endometriosis.

A 32-year-old female with uterine cervical cancer (stage Ib1) had undergone a fertility-preserving radical hysterectomy for 6 years. In the follow-up examination, no positive findings were indicated, even if she had complained about dysmenorrhea for 2 years before the second

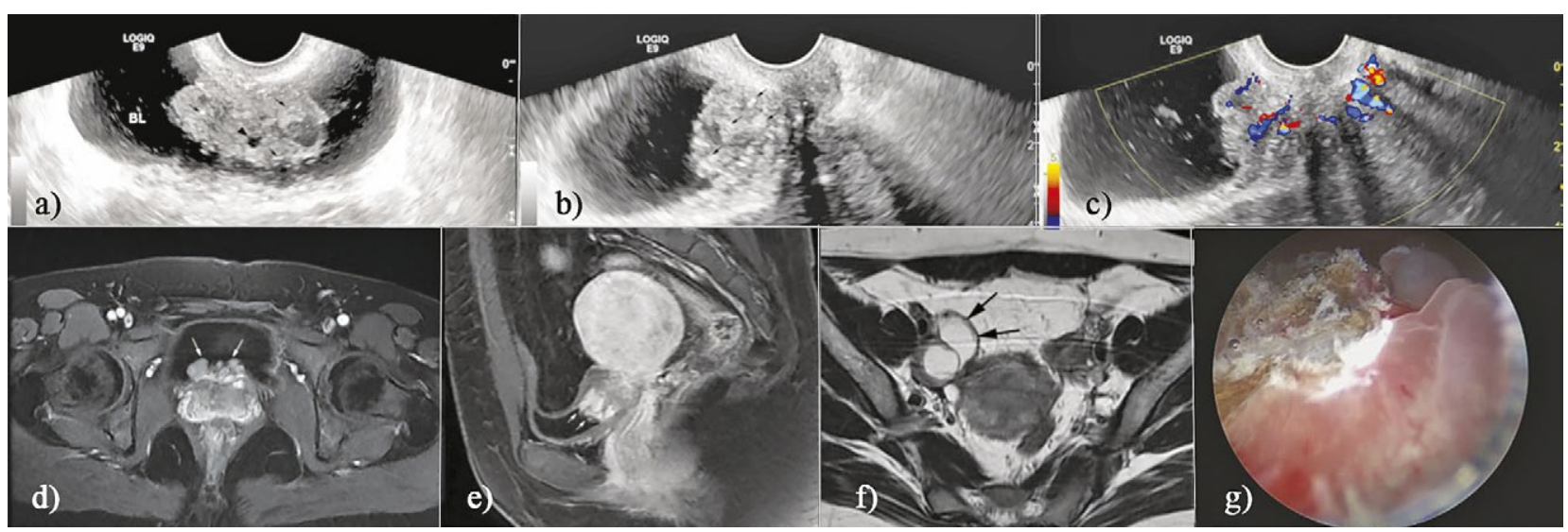

Fig 1. Transverse (a) and longitudinal (b) transvaginal ultrasound exhibit an isoechoic solid mass measuring $4 \mathrm{~cm}$ in size, with multiple intralesional echogenic foci (arrows) and cysts (arrowhead); color Doppler ultrasound (c) shows discrete vascularity; axial (d) and sagittal (e) fat-suppressed T2W and T1W images demonstrate a lobulated polypoid mass with hyperintensity located in the trigone area of bladder (arrows) and axial T1W image (f) shows ovarian cysts(arrows) with hyperintensity, which prove to be ovarian endometriomas; $(\mathrm{g})$ cystoscopic examination of the gross lesion.

Received 13.09.2019 Accepted 24.10.2019

Med Ultrason

2019, Vol. 21, No 4, 497-498, DOI: 10.11152/mu-2153,

Corresponding author: Yu Xia

Ultrasound Department, Peking Union Medical College Hospital, Chinese Academy of Medical Sciences and Peking Union Medical College, Shuaifuyuan 1\#, Dongcheng District, 100730 Beijing, China

E-mail: xiayupumch@126.com admission. Recently, the PET/CT at another unit showed that she may have suffered a relapse for the increased fludeoxyglucose (FDG) uptake on the vaginal apex and subsequently she was hospitalized in our center. TVU showed a solid mass, located in the trigone of the urinary bladder, with intralesional cysts and echogenic foci (fig 1a-b). Color Doppler ultrasound displayed discrete vascular flow from the base to the dome of the lesion 
(fig 1c). Magnetic resonance imaging (MRI) also identified a polypoid mass projecting into the bladder lumen with hyper-signal-intensity on fat-suppressed and nonfat-suppressed T1-and T2-weighted images (fig 1d-e). The patient was suspected to have a recurrence of cervical cancer, but the diagnosis of endometriosis could not be ruled out from the findings of the TVU. Therefore, transurethral resection of the bladder tumor (fig $1 \mathrm{~g}$ ) combined with hysteroscopy was performed and surprisingly, the histopathology revealed bladder endometriosis accompanied by cystitis glandularis.

Endometriosis involving urinary tract, mostly bladder, is an unusual condition compared with its ovarian and superficial peritoneal counterparts [2]. Cystitis glandularis is a benign pathology which could result from chronic irritation and inflammation of the bladder wall. When the two disease coexist, both the mucosal and serosal surface of bladder wall are interrupted by the lesion, thus mimicking an appearance of malignancy on imaging. In such confusing scenario, TVU can assist in differential considerations. Firstly, on ultrasound, bladder endometriosis often has internal echogenic foci, representing blood, and cystitis glandularis frequently presents with cystic change [3]. In contrast, the pelvic recurrence of uterine cancer predominantly manifests as hypoechoic solid masses, rarely showing cystic change [4]. Then, bladder endometriosis attaches closely to the anterior aspect of uterus, typically located posteriorly in the bladder. Moreover, bladder endometriosis is barely isolated and it is pivotal to search for other pelvic foci of endometriosis [2].

This case illustrates a rare bladder pathology that misguided radiologists and TVUS may help to diagnose and guide appropriate management.

\section{References}

1. Brătilă E, Comandasu DE, Coroleucă C, et al. Diagnosis of endometriotic lesions by sonovaginography with ultrasound gel. Med Ultrason 2016;18:469-474.

2. Leone Roberti Maggiore U, Ferrero S, Candiani M, Somigliana E, Viganò P, Vercellini P. Bladder Endometriosis: A Systematic Review of Pathogenesis, Diagnosis, Treatment, Impact on Fertility, and Risk of Malignant Transformation. Eur Urol 2017;71:790-807.

3. Huang Y, Liu D, Jiang L, Liu S. Assessment of Cystitis Glandularis by Transvaginal Sonography in Women. J Ultrasound Med 2012;31:1025-1031,

4. Savelli L, Testa AC, Ferrandina G, et al. Pelvic relapses of uterine neoplasms: transvaginal sonographic and Doppler features. Gynecol Oncol 2004;93:441-445.

\section{Is there a place for A-scan mode in modern eye ultrasonography?}

\section{Samoila Ovidiu}

Ophthalmology Department, "Iuliu Hatieganu” Medicine and Pharmacy University, Cluj-Napoca, Romania

\section{To the Editor,}

Modern ultrasonography (US) is focused on high resolution, 3D reconstruction, Doppler images, Duplex or Enhanced Contrast US. The A-scan mode, an US technique almost a century old, would seem to retain only

Received 19.08.2019 Accepted 28.09.2019

Med Ultrason

2019, Vol. 21, No 4, 498-499, DOI: 10.11152/mu-2157,

Corresponding author: Samoila Ovidiu

Ophthalmology Department, "Iuliu Hatieganu" Medicine and Pharmacy University, Cluj-Napoca

3-5 Clinicilor Street, Cluj-Napoca, Romania

E-mail: iovidius@yahoo.com

Phone: +40726386832

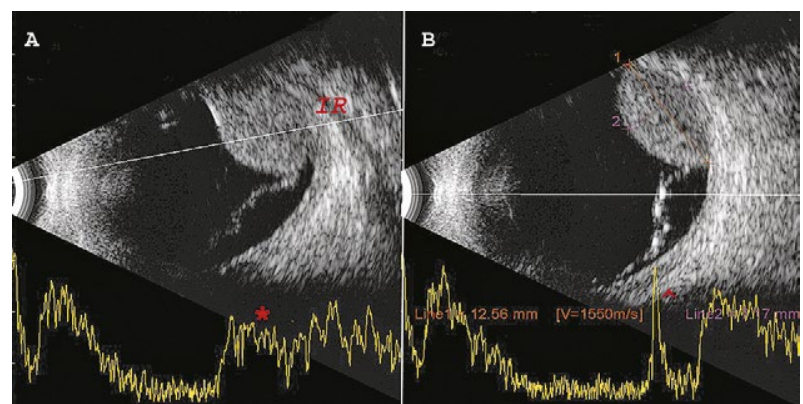

Fig 1. A. Cilliary body melanoma, in the nasal sector of the eye, close to the internal rectus (IR) insertion. A-scan shows medium reflectivity and sound attenuation $(*)$; B. Same patient; the tumor measures $12 / 7 \mathrm{~mm}$. Secondary retinal detachment is noticed; A-scan showing high amplitude wave $\left({ }^{\wedge}\right)$ 
a historical value. However, the eye anatomy with very distinct tissue borders compels us to keep the A-scan as a valuable and valid US technique.

The A-scan probe is standard equipment on all eye designated ultrasonic machines. The $8 \mathrm{MHz}$ transducer is a dedicated A-scan probe and the $10-15 \mathrm{MHz}$ transducer is a combined $\mathrm{B}$-mode and A-mode probe [1]. A-scan mode is employed in many measurements of the eye, corneal thickness (pachimetry) or axial length (biometry). All modern cataract surgeries rely on almost perfect axial measurements. The diagnostic value of the A-scan is seen in conjunction with a B-scan. The B-scan in eye US offers a rather qualitative consideration of the tissues or pathology, in terms of localization, form or homogeneity. The A-scan adds quantitative information, in terms of structure (regular or irregular), sound attenuation and probably the most important, of internal reflectivity (low, medium or high reflectivity) [2]. The A-scan can make the difference between vitreal and retinal detachment, both seen as membrane detachments on B-scans. Retinal detachment yields a much higher reflectivity on the Ascan in comparison to the vitreal detachment. The A-scan helps to differentiate various types of uveal tumors: malignant melanoma, metastasis or hemangioma, all seen as dome shaped lesions on the B-scan [3]. Characteristics for uveal melanomas on the A-scan, are medium reflectivity, high attenuation of the ultrasound and regular internal structure (fig 1).

Taking into consideration that the eye is an organ where biopsies are not usually performed, the combination of the A-scan with the B-scan, together with fluorescein angiography remains a powerful diagnostic tool. The rate of misdiagnosis and unnecessary enucleation is about $1 \%$ [4].

\section{References}

1. Silverman RH. Focused ultrasound in ophthalmology. Clin Ophthalmol 2016;10:1865-1875.

2. Kendall CJ, Prager TC, Cheng H, Gombos D, Tang RA, Schiffman JS. Diagnostic Ophthalmic Ultrasound for Radiologists. Neuroimaging Clin N Am 2015;25:327-365.

3. Campagnoli TR, Medina CA, Singh AD. Choroidal melanoma initially treated as hemangioma: diagnostic and therapeutic considerations. Retin Cases Brief Rep 2016;10:175182.

4. Mithal KN, Thakkar HH, Tyagi MA, Bharwada RM, Billore PO. Role of echography in diagnostic dilemma in choroidal masses. Indian J Ophthalmol 2014;62:167.

\title{
Ultrasound use to diagnose septal panniculitis
}

\author{
Abdulhadi Jfri ${ }^{1}$, Antonio Martorell ${ }^{2}$
}

${ }^{1}$ Division of Dermatology, McGill University Health Centre, Canada, ${ }^{2}$ Division of Dermatology, Hospital of Manises, Valencia, Spain

\section{To the Editor,}

A 53-year-old-woman with a history of chronic plaque psoriasis, psoriatic arthritis, hidradenitis suppurativa, fibromyalgia and cutaneous lupus was previously on biologics therapy including infliximab, etanercept,

Received 14.09.2019 Accepted 20.10.2019

Med Ultrason

2019, Vol. 21, No 4, 499-500, DOI: 10.11152/mu-2203,

Corresponding author: Abdulhadi Jfri, MD

Division of Dermatology, McGill University

Health Centre, Rm. L8-201, 1650 Cedar Avenue

Montreal, QC H3G 1A4, Canada

E-mail: Abdulhadi.jfri@mail.mcgill.ca ustekinumab, adalimumab, certolizumab, golimumab and secukinumab. Six months of subcutaneous pre-fill autoinjector pen of ixekizumab (anti-IL17) $80-\mathrm{mg} / \mathrm{ml}$ every 4 weeks, alternating between arms, abdomen and thighs, provided overall improvement of her psoriatic arthritis and moderate-to-severe psoriasis. Two abdominal injections produced redness at the site: we switched to a syringe, but the pharmacy inadvertently delivered the pen and she injected her abdomen. A week later she experienced progressive left lower abdomen redness and pain. Examination revealed an indurated tender erythematous large plaque (fig 1a). The differential diagnosis included cellulitis. An ultrasound was performed and showed 


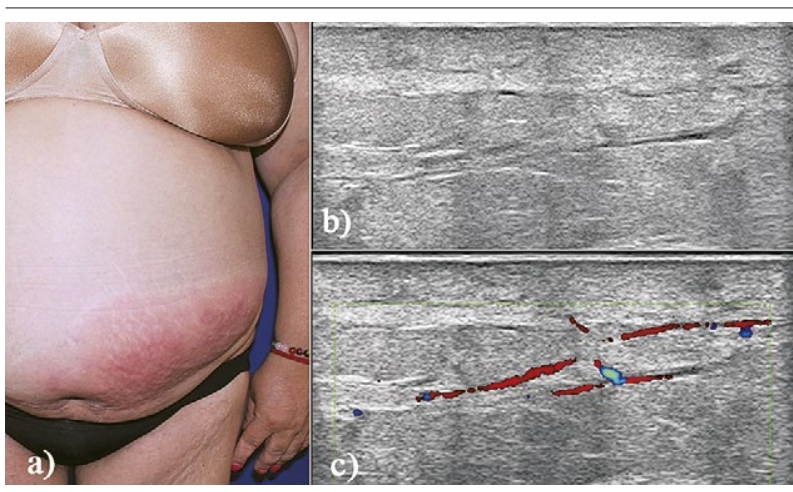

Fig 1. Left lower abdomen indurated erythematous large plaque (a). Ultrasound showed septal thickening with hypoechogenicity (b) and increased septal Doppler flow (c).

septal thickening with hypoechogenicity (fig 1b) with increased septal Doppler flow (fig 1c), consistent with secondary ixekizumab autoinjector panniculitis.

Ixekizumabis a new humanized monoclonal antibody (IgG) that targetsinterleukin 17A (IL-17A) and is used more now to treat patients with moderate to severe plaque psoriasis [1]. Ixekizumab is administered subcutaneously. There are two ways to administer the medication via autoinjector pen or syringe [2].
Our patient was treated with a single $0.5 \mathrm{ml}$ of $40-\mathrm{mg} /$ $\mathrm{ml}$ intramuscular triamcinolone injection and switched to a syringe, which controlled this phenomenon without the need of antibiotics and allowed her to continue with antiIL-17 ixekizumab. Skin biopsies for histology and tissue culture are unnecessary in the presence of fast, safe and non-invasive ultrasound. Other treatments to consider include oral prednisone or anti-inflammatory antibiotics such as tetracyclines [3].

\section{References}

1. Paul C, Griffiths CEM, van de Kerkhof PCM, et al. Ixekizumab provides superior efficacy compared with ustekinumab over 52 weeks of treatment: Results from IXORAS, a phase 3 study. J Am Acad Dermatol 2019;80:70-79. e3.

2. Deodhar A, Poddubnyy D, Pacheco-Tena C, et al. Efficacy and Safety of Ixekizumab in the Treatment of Radiographic Axial Spondyloarthritis: Sixteen-Week Results From a Phase III Randomized, Double-Blind, Placebo-Controlled Trial in Patients With Prior Inadequate Response to or Intolerance of Tumor Necrosis Factor Inhibitors. Arthritis Rheumatol 2019;71:599-611.

3. Requena L, Yus ES. Panniculitis. Part I. Mostly septal panniculitis. J Am Acad Dermatol 2001;45:163-183.

\title{
Lung ultrasound in the diagnosis of radio-occult pneumonia in the returned traveller: a case of measles pneumonia
}

\author{
Cleide Luís das Neves Rato Barrigoto', YaleTung-Chen², Patricia Suárez-García ${ }^{3}$, Tomás \\ Villén-Villegas ${ }^{2}$, Fernando de la Calle-Prieto ${ }^{4}$
}

${ }^{1}$ Internal Medicine Department. Hospital de São José, Centro Hospitalar Lisboa Central, Lisbon, Portugal, ${ }^{2}$ Servicio de Urgencias. Hospital Universitario La Paz, Madrid, Spain, ${ }^{3}$ Complejo Hospitalario Universitario A Coruña, Servicio de Atención Primaria y Comunitaria, A Coruña, Spain, ${ }^{4}$ Unidad de Enfermedades Tropicales, Hospital Universitario La Paz, Madrid, Spain

Received 08.09.2019 Accepted 03.11.2019 Med Ultrason

2019, Vol. 21, No 4, 500-501, DOI: 10.11152/mu-2196,

Corresponding author: Yale Tung-Chen

Servicio de Urgencias,

Hospital Universitario La Paz,

Paseo de la Castellana, 261.

28046 Madrid, Spain

E-mail: yale.tung@salud.madrid.org

\section{To the Editor,}

Pneumonia is a severe complication that accounts for most measles-associated deaths [1], produced by the measles virus $(\mathrm{MeV})$ alone or secondary to Adenovirus, Herpes Simplex Virus or bacterial superinfection. The diagnosis is suggested by clinical manifestations and is confirmed by high serum levels of measles-specific IgM, 
nasopharyngeal swab culture or urine culture. Treatment is supportive but, when respiratory complications are present, hospitalization should be considered [2].

We present the case of a previously healthy 40 -yearold man presented to the emergency department with a fever and erythematous rash, 7 days after returning from a trip to Thailand. The rash started on the $5^{\text {th }}$ day of fever and progressed from head to toes with predominance of lesions in the chest. Cough, conjunctivitis and jugal lesions compatible with Koplik spots were also present. No pre-travel consultation was received, therefore specific vaccines were not given. The diagnosis of measles was made by the typical clinical presentation and it was confirmed by serologic testing (IgM positive/IgG negative). Laboratory results showed no remarkable results. The physical exam revealed a mild respiratory distress, with oxygen saturation of $95 \%$ while breathing room air. Both lung auscultation and chest X-ray were normal. Lung ultrasound (LUS) was performed using a GE LOGIQ-e ultrasound system, with a linear transducer (5$10 \mathrm{MHz}$ ) (General Electrics Healthcare, Madrid, Spain), showing bilateral subpleural consolidations and diffuse B-lines (fig 1), interpreted as measles-associated pneumonia. The patient was admitted for close monitoring, bronchodilators and oxygen administration. The patient's condition improved, followed by the resolution of the consolidation.

Measles cases and outbreaks are increasing in the Western countries, catalyzed by vaccine hesitancy and growing as a global threat [3]. In this clinical case, the patient had recently been in Thailand where the prevalence of measles is high [3]. Although the recent travel could suggest a traveler disease, the clinical features were pathognomonic of measles.

Measles associated pneumonia is a severe complication and the main cause of death in these patients, therefore an early diagnosis is imperative. However, due to low sensibility of physical examination and chest X-ray, $\mathrm{CT}$ scan of the thorax is the gold standard for diagnosing. Nevertheless, the use of CT scans are more costly, less available and expose patients to high doses of ionizing radiation. Bedside LUS is a reliable, safe, cost-efficient

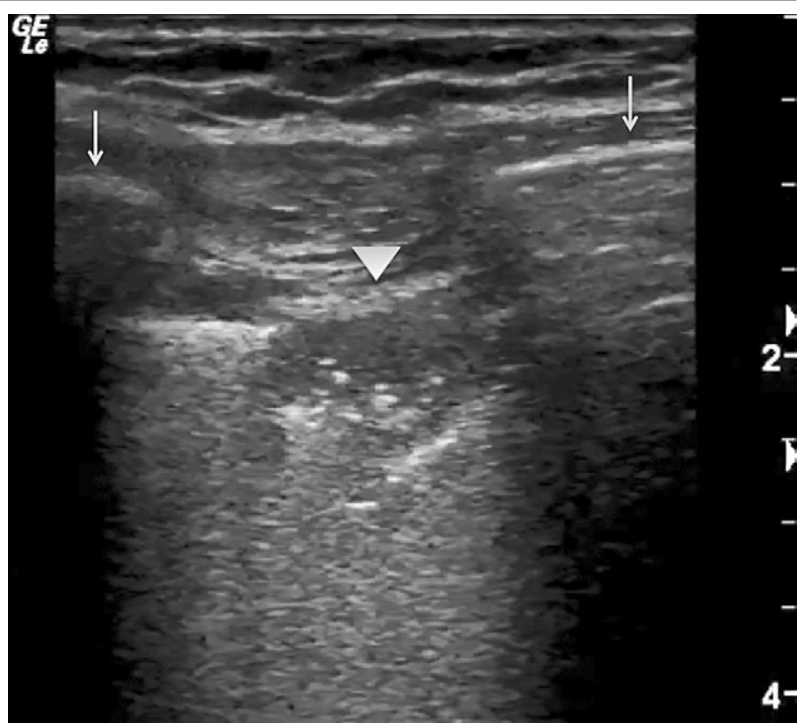

Fig 1. Longitudinal scan of the right lower lobe, posterior basal segment of the lung showing subpleural consolidation (arrowhead). Arrows: ribs

and accurate initial imaging modality in patients with shortness of breath. In our case, LUS confirmed the measles pneumonia, right at the bedside, in the setting of the normal chest X-ray. The presence of subpleural consolidations and focal B-lines is highly specific for interstitial syndrome.

In conclusion, LUS is an excellent tool in the emergency department for identifying viral pneumonia, earlier than chest X-ray and therefore may serve as a therapy guide in these clinical situations.

\section{References}

1. Perry RT, Halsey NA. The clinical significance of measles: a review. J Infect Dis 2004;189 Suppl 1:S4-S16.

2. Basile V, Di Mauro A, Scalini E, et al. Lung ultrasound: a useful tool in diagnosis and management of bronchiolitis. BMC Pediatr 2015; 15:63.

3. Epidemiological update: Measles - monitoring European outbreaks, 15 September 2017.Available at: http://ecdc.europa.eu/en/news-events/epidemiological-update-measlesmonitoring-european-outbreaks-15-september-2017 


\title{
'Tattoo-free' physiatry irrevocable with ultrasonography
}

\author{
Levent Özçakar'1, Ahmad J. Abdulsalam² ${ }^{2}$ Vincenzo Ricci $^{3}$
}

${ }^{1}$ Hacettepe University Medical School, Department of Physical and Rehabilitation Medicine, Ankara, Turkey, ${ }^{2}$ Department of Physical Medicine and Rehabilitation, Physical Medicine and Rehabilitation Hospital, Andalous, Kuwait, ${ }^{3}$ IRCCS Rizzoli Orthopaedic Institute, Department of Biomedical and Neuromotor Science, Physical and Rehabilitation Medicine Unit, Bologna, Italy

\section{To the Editor,}

Interventions for several pathologies are commonplace in the daily practice of musculoskeletal physicians. Undoubtedly, both for precise targeting and for avoiding collateral injury, guidance is paramount during those procedures. In this sense, while palpatory anatomy has been widely used previously (blind technique), with/after the convenient utility of ultrasound (US) imaging in musculoskeletal practice, the question whether blind injections are still ethical or historical is being raised [1]. This question comprises issues not only for prompt targeting but also for precise diagnosis (as well as clinical decision making). Either due to a lack of awareness or the absence of a US machine, the number of physicians still performing blind injections is unfortunately still high.

Interestingly, or sometimes owing to the particular blind injection technique, physicians (need to) make some anatomical drawings on the patient's skin (fig 1). Again, for a physician who is routinely using US imaging and guidance, those drawings can be considered as 'hieroglyphic stone age doodles', metaphorically. Taking also into account the possibility of those palpations not always being correct (e.g. in the presence of osteophytes), such an approach can simply be deemed inappropriate, if not primitive [2]. Briefly, one could never be sure what he/she is 'missing' during a blind intervention. Apart from the aforementioned arguments, there are also reports on the infection risks related with those indelible markings [3]. We intentionally have made an ironic

Received 02.11.2019 Accepted 06.11.2019

Med Ultrason

2019, Vol. 21, No 4, 502-503, DOI: 10.11152/mu-2293,

Corresponding author: Ahmad Jasem Abdulsalam

Department of Physical Medicine and

Rehabilitation, Physical Medicine and

Rehabilitation Hospital, Andalous, Kuwait

Phone: +96597923190

E-mail: dr.ahmad.j.abdulsalam@gmail.com

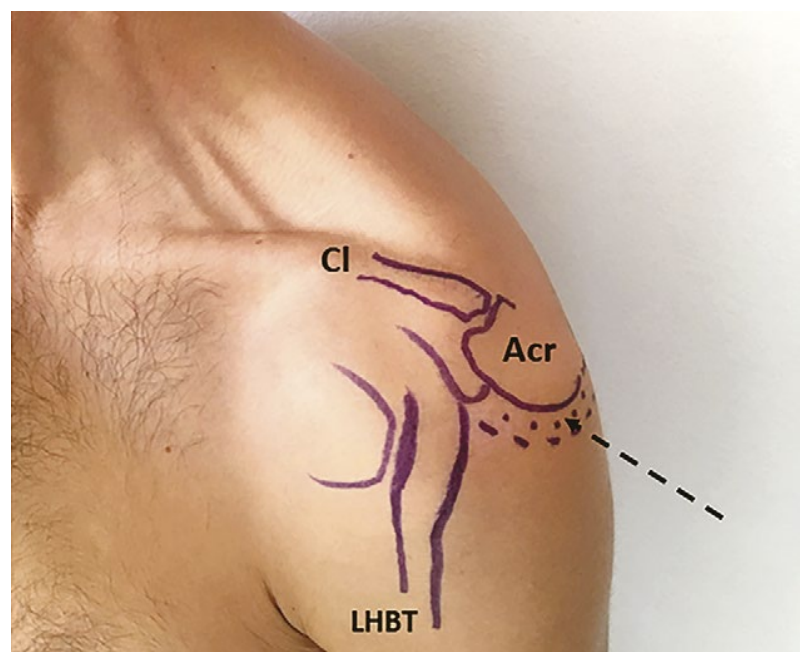

Fig 2. Skin markings on the patient before a shoulder injection. It is quite difficult to be sure about the eventual destination of the needle tip (e.g. intra-bursal, extra-bursal, intra-tendinous, intra-articular). Additionally, it is unclear what it is missing/hitting on the way. $\mathrm{Cl}$; clavicle, Acr; acromion, LHBT; long head of the biceps tendon, black dotted arrow; needle's pathway

similarity between such markings and tattoos- markings on the body with an indelible design - which have been popular from the time of ancient Egypt and their mummies, and thereafter throughout different times, cultures and civilizations [4].

Needless to say, in the era of today's technology (i.e. internet world) pigeons or smoke for communication is rarely used. Likewise, in this 'US world', physiatrists (actually musculoskeletal physicians in general) should think once again how to do their daily clinical practice. Of note, inaccessibility to a US machine should not be so discouraging since there are innumerous solutions once a decision is made to find one. In conclusion US has unconditionally found its place in our specialty; therefore, it is actually US imaging that is irrevocable in our practice not tattoos [5]. 


\section{References}

1. Özçakar L, Onat ŞŞ, Gürçay E, Kara M. Are Blind Injections Ethical or Historical?: Think Twice with Ultrasound. Am J Phys Med Rehabil 2016;95:158160.

2. Özçakar L, Kara M, Onat ŞŞ, Gürçay E. "Don’t Touch my Bone and Don't Run into my Nerve". Am J Phys Med Rehabil 2016;95:e125-e126.
3. Ballal MS, Shah N, Ballal M, O’Donoghue M, Pegg DJ. The risk of cross-infection when marking surgical patients prior to surgery - review of two types of marking pens. Ann R Coll Surg Engl 2007;89:226-228.

4. Deter-Wolf A, Robitaille B, Krutak L, Galiot S. The world's oldest tattoos. J Archaeol Sci Rep 2016;5:19-24.

5. Akkaya N, Ulaşlı AM, Özçakar L. Use of musculoskeletal ultrasound in clinical studies in physiatry: the "stethoscope" is also becoming the "pen". J Rehabil Med 2013;45:701-702. 